\title{
Large microlensing: history and perspectives
}

\author{
A. V. Byalko \\ L. D. Landau Institute for Theoretical Physics, 117940, Kosygin St., 2, Moscow, Russia \\ E-mali: byalko@landau.ac.ru
}

The recent observational confirmation [1,2] of microlensing (i.e., gravitational focusing of light from a distant star by a small invisible body) becomes a scientific event of rather unusual appearance: this phenomena was theoretically predicted by Einstein 60 years ago [3], its observational probabilities were analyzed by me [4] and later by Paczynski [5]. Being finally observed the phenomena reveals difficulties to become a valuable observational instrument [6]. Actually the instrumental capacity of microlensing and the information about lens bodies occur to be limited: observations of a single event cannot say definitely what are the mass and transverse velocity of the lensing body, and how far it is located - only the product of the mass and distance becomes an actual outcome of each microlensing observation. I will report the results of a statistical approach to brightness changes of distant objects resulting in multiple microlensing. The theoretical time-correlation function will be compared with that for quasars observations [7]. Finally another observational procedure will be briefly discussed: the brightness curve of a pulsar in the radio waverange can reveal a diffraction pattern which could give information on both mass and distance to the lensing object.

PACS: 98.80.-k

1. C. Alcock et. al., Nature 365, 621 (1993).

2. E. Aubourg et. al., Nature 365, 623 (1993).

3. A. Einstein, Science 84, 506 (1936).

4. A. V. Byalko, Astronomicheskii Zh. 46, 998 (1969) (in Russian), the English translation is published in 30 Years of the Landay Institute. Selected papers, I. M. Khalatnikov and V. P. Mineev (eds.), World Scientific (1996).

5. B. Paczynski, Astrophys. J. 304, 1 (1984).

6. A. V. Byalko, Microlensing: Statistical Approach, Astronomical and Astrophysical Trans. 10, 177 (1995); Priroda 24 (1994) (in Russian).

7. M. R. S. Hawkins, Nature 366, 242 (1993). 